\title{
SPECTRAL APPROXIMATIONS OF A NORMAL OPERATOR
}

\author{
RICHARD BOULDIN
}

\begin{abstract}
If $\Lambda$ is a closed convex set in the complex plane then $\Re(\Lambda ; H)$ denotes all the normal (bounded linear) operators on the fixed separable Hilbert space $H$ with spectrum contained in $\Lambda$. The fixed operator $A$ has $N$ as an $\varkappa(\Lambda ; H)$-approximant provided $N$ belongs to $\Re(\Lambda ; H)$ and the operator norm $\|A-N\|$ equals $\rho_{\Lambda}(A)$, the distance from $A$ to $\mathscr{\imath}(\Lambda ; H)$. With some hypothesis on $\Lambda$, this note proves that the dimension of the convex set of all $\Re(\Lambda ; H)$-approximants of normal operator $A$ is $\left(\operatorname{dim} H_{0}\right)^{2}$ where $H_{0}$ is the orthogonal complement of $\operatorname{ker}\left(|A-F(A)|-\rho_{\Lambda}(A)\right)$ and $F(z)$ is the unique distaince minimizing retract of the complex plane onto $\Lambda$.
\end{abstract}

1. Introduction. If $\Lambda$ is a closed convex set in the complex plane then $\Re(\Lambda$; $H$ ) denotes all the normal (bounded linear) operators on the fixed separable Hilbert space $H$ with spectrum contained in $\Lambda$. The fixed operator $A$ has $N$ as an $\Re(\Lambda ; H)$-approximant provided $N$ belongs to $\Re(\Lambda ; H)$ and the operator norm $\|A-N\|$ equals $\rho_{\Lambda}(A)$, the distance from $A$ to $\Re(\Lambda ; H)$. The set of all $\Re(\Lambda ; H)$-approximants of $A$ is denoted $\Re(\Lambda ; A)$. Provided $A$ is normal and $\Lambda$ is a closed convex subset of some straight line in the complex plane, the main theorem of this note constructs enough members of $\Re(\Lambda ; A)$ to determine its dimension as a convex set (see [7, pp. 7-9]). Thus, the main theorem generalizes previously known results for positive approximants [1, Theorem 5.2] and selfadjoint approximants [2, Corollary 3.3]; it also answers analogous questions for best approximation by selfadjoint and nonnegative contractions. Halmos [4] suggests consideration of nonnegative contractions.

It appears that the proof of the main theorem has isolated the precise ingredients for establishing such results. Perhaps greater clarity is a consequence. To eliminate trivialities it is assumed, henceforth, that $\Lambda$ contains at least two points.

2. Preliminaries. Before the main theorem is proved, consideration is given to the hypothesis which is responsible for limiting $\Lambda$. The appropriateness of this hypothesis will be clear from the proof of the main theorem. If $Q$ is some orthogonal projection on $H$, then $Q T \mid Q H$ is the compression of $T$ to $Q H$.

2.1. LEMMA. If $\operatorname{dim} H \geqslant 3$ then the following conditions on the closed convex set $\Lambda$ are equivalent:

(i) $\Lambda$ is contained in some straight line in the complex plane,

(ii) $\Re(\Lambda ; H)$ is closed under the formation of compressions, and

(iii) $\Re(\Lambda ; H)$ is convex.

Received by the editors September 13, 1978 and, in revised form, January 9, 1979. AMS (MOS) subject classifications (1970). Primary 47A99; Secondary 47B20. 
Proof. Suppose $\Lambda$ is not contained in some straight line. After a suitable translation and expansion, one may assume that $\{1,-1, i,-i\} \subset \Lambda$. Let $\{e, f, g\}$ be an orthonormal set and define $A$ by

$$
A=\langle\cdot, e\rangle e+\langle\cdot, f\rangle \text { if }-\langle\cdot, g\rangle \text { ig. }
$$

Clearly $A$ is normal with either $\{1, i,-i\}$ or $\{1, i,-i, 0\}$ as its spectrum $\sigma(A)$; hence, $A$ belongs to $\mathscr{T}(\Lambda ; H)$. Routine computations show that $A$ compressed to the subspace $\operatorname{span}\{e+f+g, e-f\}$ is not normal. Thus, (ii) implies (i); since the converse is trivial, (ii) is equivalent to (i).

Continuing the above assumptions, define $B$ and $C$ by

$$
B=\langle\cdot, e\rangle e-\langle\cdot, f\rangle f, \quad C=\langle\cdot, e\rangle \text { if }+\langle\cdot, f\rangle \text { ie. }
$$

Clearly $B=B^{*}, \sigma(B)=\{1,-1,0\}, C=-C^{*}, \sigma(C)=\{i,-i, 0\}$ and so $B$ and $C$ belong to $\Re(\Lambda ; H)$. Since the convex combination $\frac{1}{2} B+\frac{1}{2} C$ is not normal, it follows that (iii) implies (i). Since the converse is trivial, (iii) is equivalent to (i).

2.2. Corollary. If $\mathscr{\Re}(\Lambda ; K)$ is closed under the formation of compressions then for any Hilbert space $K$ the dimension of $\Re(\Lambda ; K)$, as a convex set, is $(\operatorname{dim} K)^{2}$.

Proof. If $\operatorname{dim} K$ is infinite then it is easy to see that the dimension of $\Re(\Lambda ; K)$ is infinite. Henceforth, $\operatorname{dim} K$ is assumed to be finite. Since $\Lambda$ is a subset of some straight line in the plane, it is easy to see that it suffices to assume $\Lambda$ is a subset of the real numbers $R$.

First it is shown that $\operatorname{dim} \Re(R ; K)$ does not exceed $(\operatorname{dim} K)^{2}$. If $\left\{e_{1}, \ldots, e_{n}\right\}$ is an orthonormal basis for $K$ then consideration of matrices shows that the following operators form a basis for $\mathscr{\ell}(R ; K)$ :

$$
\begin{gathered}
R_{j}=\left\langle\cdot, e_{j}\right\rangle e_{j}, \quad R_{k j}=\left\langle\cdot, e_{k}\right\rangle e_{j}+\left\langle\cdot, e_{j}\right\rangle e_{k}, \\
A_{k j}=\left\langle\cdot, e_{k}\right\rangle i e_{j}-\left\langle\cdot, e_{j}\right\rangle i e_{k} \quad \text { for } j, k=1, \ldots, n .
\end{gathered}
$$

There are $n$ operators like $R_{j}$, there are $(n-1) n / 2$ like $R_{k, j}$ and $(n-1) n / 2$ like $A_{k, j}$. Thus, the basis contains $n^{2}$ operators.

Let $a$ and $b$ be real numbers with $a<b$. If it is shown that $\operatorname{dim} \Re([a, b]$; $K)$ is at least $(\operatorname{dim} K)^{2}$ then one can conclude that $\operatorname{dim} \Re([a, b] ; K)$ equals $(\operatorname{dim} K)^{2}$. Thus, it suffices to note that the following operators form a linearly independent set in $\Re([a, b] ; K)$ :

$$
\begin{aligned}
P_{j} & =\left\langle\cdot, e_{j}\right\rangle e_{j}(a+b) / 2, \\
P_{k, j} & =\left\langle\cdot, e_{k}\right\rangle e_{j}(b-a) / 2+\left\langle\cdot, e_{j}\right\rangle e_{k}(b-a) / 2+I(a+b) / 2, \\
Q_{k, j} & =\left\langle\cdot, e_{k}\right\rangle i e_{j}(b-a) / 2-\left\langle\cdot, e_{j}\right\rangle i e_{k}(b-a) / 2+I(a+b) / 2 .
\end{aligned}
$$

There are $n$ operators like $P_{j}$ and $n(n-1)$ operators like either $P_{k, j}$ or $Q_{k, j}$.

The cases that $a$ is $-\infty$ or $b$ is $\infty$ are handled in a manner analogous to the argument of the preceding paragraph. 
3. Main results. In the proof of the main theorem several subspaces will be described in terms of the spectral measure of the normal operator $A$. Since the spectral measure is difficult to construct in concrete cases, an alternative description of the most important subspace is developed.

P. R. Halmos constructed an $\Re(\Lambda ; H)$-approximant for a normal operator $A$ in [5]. If $F(z)$ is the unique distance minimizing retract of the complex plane onto $\Lambda$ then the Halmos $\Re(\Lambda ; H)$-approximant of $A$ is $F(A)$. Since $F(z)$ is continuous, the operational calculus for $A$ defines $F(A)$ and shows the distance $\rho_{\Lambda}(A)$ to be $\sup \{\operatorname{dist}(z, \Lambda): \mathrm{z} \in \sigma(A)\}$ where $\sigma(A)$ is the spectrum of $A$ and $\operatorname{dist}(z, \Lambda)$ is $\inf \{|z-w|: w \in \Lambda\}$. The compact set

$$
\left\{z: \operatorname{dist}(z, \Lambda)=\rho_{\Lambda}(A) \text { and }|z|<\|A\|\right\}
$$

will be denoted by $\Gamma$.

3.1. LEMMA. Let $E(\cdot)$ be the spectral measure for the normal operator $A$; let $\rho_{\Lambda}(A), F(z)$ and $\Gamma$ be described by the preceding paragraph. Then one has

$$
\operatorname{ker}\left(|A-F(A)|-\rho_{\Lambda}(A)\right)=E(\Gamma) H .
$$

Proof. Since $\rho_{\Lambda}(A)^{2}-|A-F(A)|^{2}$ is nonnegative, $f$ belongs to the kernel of that operator if and only if

$$
\left\langle\left(\rho_{\Lambda}(A)^{2}-|A-F(A)|^{2}\right) f, f\right\rangle=0
$$

which is equivalent to

$$
\int\left(\rho_{\Lambda}(A)^{2}-|z-F(z)|^{2}\right) d\langle E(z) f, f\rangle=0 .
$$

This last equation is equivalent to the statement that $\rho_{\Lambda}(A)=|z-F(z)|$ almost everywhere with respect to the positive measure $\langle E(\cdot) f, f\rangle$. By the definition of $\Gamma$, this holds exactly when $z$ belongs to $\Gamma$ and so (*) is equivalent to

$$
\left\langle E\left(\Gamma^{c}\right) f, f\right\rangle=0 \text { or } E\left(\Gamma^{c}\right) f=0 \text { or } f=E(\Gamma) f \in(\Gamma) H .
$$

This proves that $E(\Gamma) H$ equals the kernel of $\left(\rho_{\Lambda}(A)^{2}-|A-F(A)|^{2}\right)$. The facts that $|A-F(A)|$ is a nonnegative operator, $\rho_{\Lambda}(A)$ is a nonnegative number and

$$
\rho_{\Lambda}(A)^{2}-|A-F(A)|^{2}=\left(\rho_{\Lambda}(A)-|A-F(A)|\right)\left(\rho_{\Lambda}(A)+|A-F(A)|\right)
$$

prove the lemma.

Recall that the set of all $\Re(\Lambda ; H)$-approximants of $T$ is denoted $\Re(\Lambda ; T)$.

3.2. Lemma. Let $A$ be a normal operator and let $\Re(\Lambda ; H)$ be closed under formation of compressions. If $N$ belongs to $\Re(\Lambda ; A)$ then $E(\Gamma) H$ reduces $N$ to $F(A) \mid E(\Gamma) H$.

Proof. The following computation shows that $E(\Gamma) N \mid E(\Gamma) H$ belongs to $\Re(\Lambda ; A \mid E(\Gamma) H)$ : 


$$
\begin{aligned}
\|A|E(\Gamma) H-E(\Gamma) N| E(\Gamma) H\| & =\|E(\Gamma)(A-N) E(\Gamma)\| \\
& <\|A-N\|=\rho_{\Lambda}(A)
\end{aligned}
$$

and

$$
\begin{aligned}
\rho_{\Lambda}(A \mid E(\Gamma) H) & =\sup \{\operatorname{dist}(z, \Lambda): z \in \sigma(A \mid E(\Gamma) H)\} \\
& =\sup \{\operatorname{dist}(z, \Lambda): z \in \sigma(A) \cap \Gamma\}=\rho_{\Lambda}(A) .
\end{aligned}
$$

In the trivial case that $E(\Gamma) H=\{0\}$ the desired conclusion is trivial.

By [3, Theorem 4] the unique $\mathscr{N}(\Lambda ; H)$-approximant of $A \mid E(\Gamma) H$ is $F(A \mid E(\Gamma) H)=F(A) \mid E(\Gamma) H$. Consequently, relative to the decomposition

$$
H=E(\Gamma) H \oplus E\left(\Gamma^{c}\right) H,
$$

$N$ has a matrix of the form

$$
\left(\begin{array}{cc}
F(A) \mid E(\Gamma) H & E_{2} \\
E_{1} & E_{3}
\end{array}\right)
$$

For any unit vector $f \in E(\Gamma) H$ one has

$$
\|(A-N) f\|^{2}=\|(A-F(A)) f\|^{2}+\left\|E_{1} f\right\|^{2}
$$

and

$$
\begin{aligned}
\|(A-F(A)) f\| & >\inf \{|z|: z \in \sigma(A-F(A) \mid E(\Gamma) H)\} \\
& =\inf \{|z-F(z)|: z \in \sigma(A) \cap \Gamma\}=\rho_{\Lambda}(A) .
\end{aligned}
$$

This implies that $\left\|E_{1} f\right\|^{2}=0$ and so $E_{1}=0$. The same argument applied to $(A-N)^{*}$ shows that $E_{2}=0$ and the desired conclusion follows.

3.3. Lemma. Let $A$ be a normal operator and let $H_{0}$ be the orthogonal complement of $\operatorname{ker}\left(|A-F(A)|-\rho_{\Lambda}(A)\right)$. If $\Re(\Lambda ; H)$ is closed under the formation of compressions then the real dimension of $\Re(\Lambda ; A)$, as a convex set, does not exceed $\left(\operatorname{dim} H_{0}\right)^{2}$.

Proof. From Lemma 3.1 it follows that $H_{0}=E\left(\Gamma^{c}\right) H$ where $E(\cdot)$ is the spectral measure for $A$. From Lemma 3.2 it follows that each member of $\Re(\Lambda ; A)$ belongs to the set

$$
\left\{F(A) \mid\left(H_{0}\right)^{\perp} \oplus R: R \in \mathcal{T}\left(\Lambda ; H_{0}\right)\right\} ;
$$

this set has the same dimension as $\Re\left(\Lambda ; H_{0}\right)$ and the dimension of that is given by Lemma 2.2 .

3.4. THEOREM. Let $A$ be a normal operator and let $H_{0}$ be the orthogonal complement of $\operatorname{ker}\left(|A-F(A)|-\rho_{\Lambda}(A)\right)$. If $\Lambda$ is a closed convex set such that $\Re(\Lambda ; H)$ is closed under formation of compressions then the real dimension of the convex set $\mathscr{N}(\Lambda ; A)$ equals $\left(\operatorname{dim} H_{0}\right)^{2}$.

Proof. In view of Lemma 3.3 it suffices to show that $\operatorname{dim} \vartheta(\Lambda ; A)$ is infinite when $\operatorname{dim} H_{0}$ is infinite and that

$$
\operatorname{dim} \Re(\Lambda ; A) \geqslant\left(\operatorname{dim} H_{0}\right)^{2}
$$


holds when $\operatorname{dim} H_{0}$ is finite. Since the last inequality is easier, it is proved first.

If $\operatorname{dim} H_{0}=0$ then $H=E(\Gamma) H$ or $\sigma(A) \subset \Gamma$ it follows from [3, Theorem 4] that $F(A)$ is the unique $\Re(\Lambda ; H)$-approximant of $A$. Assume $\operatorname{dim} H_{0}$ is a positive integer and note that $\sigma\left(A \mid E\left(\Gamma^{c}\right) H\right)$ consists of a finite collection of eigenvalues. Consequently,

$$
\eta \equiv \sup \left\{\operatorname{dist}(z, \Lambda): z \in \sigma\left(A \mid E\left(\Gamma^{c}\right) H\right)\right\}
$$

is less than $\rho_{\Lambda}(A)$. For example, assume that $0 \in \Lambda$. Let $\Lambda_{0}$ be a closed convex subset of $\Lambda$ such that $0 \in \Lambda_{0}$ and if $\lambda$ is a nonzero extreme point of $\Lambda_{0}$, that is $\lambda \in \operatorname{Ext} \Lambda_{0}$, then there is some $\mu \in \operatorname{Ext} \Lambda$ with $|\lambda-\mu|=\delta$ where

$$
2 \delta=\min \left\{\rho_{\Lambda}(A)-\eta,|z| / 3: z \in \operatorname{Ext} \Lambda, z \neq 0\right\} .
$$

Let $N$ be any operator from $\Re\left(\Lambda_{0} ; H\right)$ which is reduced to zero by $E(\Gamma) H$ and $\|N\|<\delta$. Let $G(z)$ be the distance minimizing retract onto $\Lambda_{0}$. The following computation shows that

$$
T \equiv(N+F(A))|E(\Gamma) H \oplus(N+G(A))| E\left(\Gamma^{c}\right) H
$$

is in $\Re(\Lambda ; A)$.

$$
\begin{aligned}
\|A-T\| & =\max \left\{\left\|(A-F(A))|E(\Gamma) H\|,\|(A-G(A)-N)| E\left(\Gamma^{c}\right) H\right\|\right\} \\
& =\max \left\{\rho_{\Lambda}(A),\left\|(A-G(A)) \mid E\left(\Gamma^{c}\right) H\right\|+\|N\|\right\}=\rho_{\Lambda}(A) .
\end{aligned}
$$

Note that the spectral mapping theorem shows that the spectral radius of $(A-G(A)) \mid E\left(\Gamma^{c}\right) H$ does not exceed $\eta+\delta$ and since this operator is normal, its spectral radius equals its norm. This proves that $\left\{R-\left(F(A) \mid H_{0}^{\perp} \oplus\right.\right.$ $\left.\left.G(A) \mid H_{0}\right) ; R \in \Re(\Lambda ; A)\right\}$ contains a copy of $\mathscr{N}\left(\Lambda_{0}, H_{0}\right)$ and inequality (*) follows from Lemma 2.2 .

If $\operatorname{dim} H_{0}$ is infinite and $A \mid H_{0}$ has infinitely many eigenvalues (counted according to multiplicity) then the method of the preceding paragraph shows that $\operatorname{dim} \Re(\Lambda ; A)$ is infinite.

The remaining case that $\operatorname{dim} H_{0}$ is infinite and $A \mid H_{0}$ has only finitely many eigenvalues (counted by multiplicity) is the most difficult. A well-known measure theory technique for the complex plane is required, and the version presented in [6, pp. 51-54] will be used. For the reader's convenience the key ideas of that technique are appropriately reformulated. If $w$ is a complex number and $\delta$ is a positive number then the set $\{z$ : $\alpha<$ re $z<\alpha+\delta$, $\beta<\operatorname{im} z<\beta+\delta\}$ is the $\delta$-box with corner at $\alpha+i \beta$, where $\alpha$ and $\beta$ are real. For $n=1,2, \ldots$ let $S_{n}$ be the set of all $\left(2^{-n}\right)$-boxes with corners at $x+i y$ where $x$ and $y$ are both integral multiples of $2^{-n}$. Let $T_{n}$ be $\left\{C \in S_{n}\right.$ : $E(C) \neq 0\}$ where $E(\cdot)$ is the spectral measure for $A$ and let $\mathcal{T}$ be $\cup\left\{T_{1}, T_{2}, \ldots\right\}$. Take a sequence $\left\{D_{1}, D_{2}, \ldots\right\}$ from $\mathcal{T}$ such that $i>j$ implies $D_{i} \subset D_{j}$ and $D_{i} \neq D_{j}$. It is straightforward to see that $\cap\left\{D_{1}, D_{2}, \ldots\right\}$ consists of exactly one complex number, say $w$.

Because the orthogonal complement in $E\left(\Gamma^{c}\right) H$ of the span of the eigenspaces corresponding to eigenvalues of $N$ off $\Gamma$ must be infinite dimensional, 
one can assume that $N$ has no eigenvalues off $\Gamma$. Thus, with no loss of generality, it is assumed that $E(\cdot)$ has no point masses in $\Gamma^{c}$. If

$$
\delta_{k}=\left\{z: \operatorname{dist}(z, \Lambda)<\rho_{\Lambda}(A)(1-1 / k)\right\}
$$

then for $k$ sufficiently large $E\left(\Im_{k}\right)$ is not zero. Fix such a choice of $k$. If $D$ is some box from $\mathcal{S}_{k} \cap T_{n}$ then for arbitrarily large $i$ there are at least two boxes in $T_{n+i}$, say $F_{1}$ and $F_{2}$, such that $D \supset F_{1} \cup F_{2}$. Otherwise, one would obtain a finite collection of boxes $\left\{E_{1}, \ldots, E_{l}\right\}$ with the property that associated with each $E_{j}$ is a strictly decreasing sequence of boxes $\left\{E_{j, 2}, E_{j, 3}, \ldots\right\}$ such that $E\left(E_{j}\right)=E\left(E_{j, 2}\right)=E\left(E_{j, 3}\right)=\ldots$ If $w_{j}$ is defined by $\left\{w_{j}\right\}=\cap\left\{E_{j}, E_{j, 2}, \ldots\right\}$ then $w_{j}$ is a point mass for $E(\cdot)$ and one has a contradiction.

Now it is easy to construct an infinite collection of Borel sets $\mathfrak{N}=$ $\left\{M_{1}, M_{2}, \ldots\right\}$ such that $E\left(M_{j}\right) \neq 0$ and the closure of $M_{j}$, denoted $M_{j}^{-}$, does not intersect $\Gamma$. Choose $D_{1}$ from $\delta_{k} \cap T_{n(1)}$; choose $n(2)>n(1)$ such that two boxes of $T_{n(2)}$ are contained in $D_{1}$ and let $D_{2}$ be one of the two boxes. Choose $n(3)>n(2)$ such that two boxes of $T_{n(3)}$ are contained in $D_{2}$ and let $D_{3}$ be one of the two boxes. Continue this by induction. Define $M_{j}$ to be $D_{j} \backslash D_{j+1}$ and note that $\mathscr{N}=\left\{M_{1}, M_{2}, \ldots\right\}$ is the desired collection.

Let $\eta_{j}=\sup \left\{\operatorname{dist}(z, \Lambda): z \in M_{j}\right\}$ and note that $\eta_{j}<\rho_{\Lambda}(A)$. Choose $\rho_{j} \in \Lambda$ such that $\rho_{j} \notin F\left(M_{j}^{-}\right)$and $\sup \left\{\operatorname{dist}\left(z, \rho_{j}\right): z \in M_{j}\right\}$ does not exceed $\rho_{\Lambda}(A)$. Define $N_{j}$ to be $\rho_{j} E\left(M_{j}\right)+F(A) E\left(M_{j}^{c}\right)$. It is routine to verify that $N_{j}$ is an $\Re(\Lambda ; H)$-approximant of $A$ and that $\left\{N_{1}-F(A), N_{2}-F(A), \ldots\right\}$ is a linearly independent set. Thus, $\operatorname{dim} \mathfrak{N}(\Lambda ; A)$ is infinite and the theorem is proved.

\section{BIBLIOGRAPHY}

1. R. H. Bouldin, The convex structure of positive approximants for a given operator, Acta Sci. Math. (Szeged) 37 (1975), 177-190.

2. R. H. Bouldin and D. D. Rogers, Normal dilations and operator approximations, Acta Sci. Math. (Szeged) 39 (1977), 233-243.

3. C. K. Chui, P. W. Smith and J. D. Ward, Approximation with restricted spectra, Math. Z. 144 (1975), 289-297; ibid. 146 (1976), 291-292.

4. P. R. Halmos, Some unsolved problems of unknown depth about operators on Hilbert space, Proc. Roy. Soc. Edinburgh Sect. A 76A (1976), 67-76.

5. 51-58. Spectral approximants of normal operator, Proc. Edinburgh Math. Soc. 19 (1974),

6. W. Rudin, Real and complex analysis, McGraw-Hill, New York, 1966.

7. F. A. Valentine, Convex sets, McGraw-Hill, New York, 1964.

Department of MAThEMatics, University of Gborgia, Athens, Gborgin 30602 